\title{
Bebês pré-termo: aleitamento materno e evolução ponderal
}

\author{
Preterm infants: breastfeeding and weight gain \\ Bebes pre-termo: lactancia materna y aumento del peso
}

\author{
Anelize Helena Sassá', Kayna Trombini Schmidt", Bruna Caroline Rodrigues"'", \\ Sueli Mutsumi Tsukuda Ichisato"', Ieda Harumi Higarashi"', Sonia Silva Marcon"'I \\ 'Secretaria Municipal de Saúde de Maringá. Maringá-PR, Brasil. \\ "Universidade Paranaense, Curso de Enfermagem. Maringá-PR, Brasil. \\ '" Universidade Estadual de Maringá, Departamento de Enfermagem, \\ Programa de Pós-Graduação em Enfermagem. Maringá-PR, Brasil.
}

Submissão: 05-09-2012 Aprovação: 26-06-2014

\section{RESUMO}

O objetivo do estudo foi identificar fatores associados à prática do aleitamento materno (AM), ao ganho ponderal e ao estado nutricional de bebês pré-termos durante os seis primeiros meses de vida e verificar correlação entre essas variáveis e características maternas e neonatais. Estudo analítico, tipo coorte, realizado com 42 bebês pré-termos nascidos entre maio e outubro de 2008. A prevalência de AM foi de 40,5\% aos seis meses e a média de ganho ponderal variou entre 18 e $40 \mathrm{~g} /$ dia. A prática de $\mathrm{AM}$ esteve associada ao menor peso e à menor idade gestacional de nascimento. $\mathrm{O}$ ganho ponderal apresentou correlação positiva com a idade gestacional e o peso ao nascer, a idade materna e a prática do AM aos 15 dias após a alta. Ser prematuro não impediu a prática do AM; entretanto, o ganho ponderal foi maior entre aqueles nascidos com melhores condições de crescimento e maturação intrauterina.

Descritores: Enfermagem Materno-Infantil; Nutrição do Lactente; Prematuro; Aleitamento Materno; Crescimento.

ABSTRACT
The objective was to identify factors associated with the practice of breastfeeding (BF), weight gain and nutritional status of preterm infants during the first six months of life and verify the relationship between these variables and maternal and neonatal characteristics. Analytical studies, cohort, performed with 42 preterm infants born between May and October 2008. The prevalence of BF was $40.5 \%$ at six months and mean weight gain ranged between 18 and $40 \mathrm{~g} /$ day. The practice of BF was associated with lower weight and lower gestational age at birth. Weight gain was positively correlated with gestational age and birth weight, maternal age and the practice of breastfeeding at 15 days after discharge. Being premature has not stopped the practice of BF; however, weight gain was higher among those born with better growth and maturation in utero.

Key words: Maternal-Child Nursing; Infant Nutrition; Premature; Breastfeeding; Growth.

\section{RESUMEN}

El objetivo fue identificar los factores asociados con la práctica de la lactancia materna (LM), aumento de peso y el estado nutricional de los recién nacidos prematuros durante los primeros seis meses de vida y verificar la relación entre estas variables y las características maternas y neonatales. Estudio analítico de cohorte, realizado con 42 bebés prematuros nacidos entre mayo y octubre de 2008. La prevalencia de la LM fue de $40,5 \%$ a los seis meses y la media de aumento de peso osciló entre 18 y 40g/día. La práctica de la LM se asoció con menor peso y menor edad gestacional al nacer. El aumento de peso se correlacionó positivamente con la edad gestacional y peso al nacer, la edad materna y la práctica de la LM a los 15 días después del alta. Ser prematuro no se ha detenido la práctica de la LM, sin embargo el aumento de peso fue mayor entre los nacidos con un mejor crecimiento y la maduración en el útero.

Palabras clave: Enfermería Maternoinfantil; Nutrición del Lactante; Prematuro; Lactancia Materna; Crecimiento. 


\section{INTRODUÇÃO}

A avaliação nutricional da criança compreende, além do exame físico e anamnese, o acompanhamento de medidas antropométricas, bioquímicas e da composição corpórea. Por ser de simples obtenção e facilmente reproduzível, o peso é a medida mais utilizada na avaliação da situação nutricional e está intimamente relacionado ao crescimento da criança. Para garantir o crescimento e desenvolvimento adequados do lactente, a recomendação vigente é manter o aleitamento materno exclusivo nos primeiros seis meses de vida ${ }^{(1)}$.

O Aleitamento Materno (AM) é a forma mais natural e segura de alimentar a criança no início da vida, em razão dos diversos componentes imunológicos presentes no leite materno (LM), tornando esta prática essencial para alcançar crescimento e desenvolvimento infantis adequados, além de promover benefícios para a saúde física e psíquica da mãe e do bebê(2).

Sabe-se que lactentes alimentados com leite materno e com fórmulas diferem quanto ao crescimento físico e ao desenvolvimento cognitivo, emocional e social(2). Ademais, a lactante de pré-termo possui em seu leite concentração maior de proteína, sódio, cálcio, lipídios e particularidades anti-infecciosas ${ }^{(3)}$.

Nesse sentido, e considerando os fatores existentes no leite humano e a técnica de amamentação, ratifica-se a afirmação de que a prática do AM proporciona à criança inúmeros benefícios, dentre os quais crescimento infantil adequado, proteção contra infecções, melhor desenvolvimento da musculatura da cavidade bucal, com efeitos positivos inclusive na inteligência; e ainda, diminuição do risco de alergias, hipertensão arterial, colesterol alto e diabetes mellitus, reduzindo a chance de obesidade, entre outros ${ }^{(4)}$.

Especificamente para os bebês pré-termo, o AM pode trazer ainda mais algumas vantagens, pois as propriedades nutritivas e imunológicas do leite humano favorecem a maturação gastrintestinal, o fortalecimento do vínculo mãe-filho, aumento no desempenho neuropsicomotor, proteção antioxidante, menor incidência de infecções, menor tempo de hospitalização e menor incidência de reinternações ${ }^{(4)}$.

Nesta perspectiva e, considerando que o crescimento está diretamente relacionado à terapia nutricional instituída, o objetivo deste estudo foi identificar fatores associados à prática de AM, ao ganho ponderal e ao estado nutricional (score Z) de bebês prematuros aos 15 e 45 dias após a alta hospitalar, e aos três e seis meses de vida, assim como verificar se há correlação entre as características maternas e neonatais e a prática do aleitamento materno com o ganho ponderal e o estado nutricional (score Z) destes bebês.

\section{MÉTODO}

Estudo analítico, realizado com bebês prematuros nascidos em Maringá-PR, no período de 01 de maio a 31 de outubro de 2008. Trata-se de um recorte de estudo matricial do tipo coorte realizado com todos os recém-nascidos incluídos no Programa de Vigilância do Bebê de Risco (PVBR) do município no período em questão.
O PVBR existe no município desde 2000, e tem como propósito acompanhar bebês que apresentam fatores diversos considerados de risco, tais como: baixo peso ao nascer (peso $<2500 \mathrm{~g}$ ), pontuação Apgar menor ou igual a sete no $5^{\circ}$ minuto de vida, prematuridade (idade gestacional inferior a 37 semanas), idade materna menor que 18 anos, presença de anomalias, e outros critérios que incluem, por exemplo, ser fiIho de mãe HIV positivo, a condição socioeconômica familiar precária e/ou uso de drogas de abuso pela mãe.

A equipe de Vigilância Epidemiológica da Secretaria de Saúde do município visita todos os hospitais diariamente para o preenchimento/recolhimento das fichas de nascidos vivos. Quando identificado o nascimento de uma criança com um ou mais critérios considerados de risco, ela é incluída no PVBR, ainda durante a internação hospitalar. A ficha de inclusão no programa é preenchida em duas vias, sendo uma arquivada no Setor de Vigilância e a outra encaminhada para a unidade básica de saúde (UBS) de referência, conforme o endereço da família, de modo que o crescimento e desenvolvimento das crianças possam ser avaliados mensalmente durante o primeiro ano de vida.

O bebê prematuro cuja família aceitou participar do estudo matricial foi avaliado até os doze meses de vida, por meio de seis VD realizadas aos 15 e aos 45 dias de vida, e aos três, seis, nove e 12 meses de vida. Os bebês que, após o nascimento, permaneceram internados por mais de 30 dias (prematuridade extrema e/ou com muito baixo peso ao nascer), as primeiras VD foram realizadas levando em consideração a data da alta hospitalar, visto ser comum essas crianças permanecerem hospitalizadas por longo período.

Durante as VD foram realizadas entrevistas com a mãe, utilizando-se um questionário do tipo misto contendo perguntas referentes à família, mãe e criança. Além disso, foi realizado o exame físico, mensuração do comprimento com régua antropométrica horizontal, e verificação do peso em balança digital elétrica, com precisão de cinco gramas. Para garantir que a antropometria dos bebês e os dados fossem coletados nos períodos estipulados para avaliação ( 15 e 45 dias, três, seis, nove e 12 meses), as VD foram programadas para serem realizadas com intervalo máximo de três dias antes ou três dias depois, tendo como referência a data do nascimento da criança.

Especificamente para o presente estudo, foram utilizados os dados referentes às quatro primeiras $\mathrm{VD}$, considerando os dados de caracterização das mães e dos bebês, as informações sobre a prática do AM e a evolução ponderal das crianças. Foram excluídas da avaliação as crianças cujas fichas não apresentavam alguma dessas informações.

A evolução ponderal foi analisada calculando-se o ganho de peso diário num determinado período de tempo, o que neste estudo compreendeu os intervalos entre o nascimento e a VD realizada aos quinze dias após a alta, e os intervalos entre uma VD e outra sucessiva. Para a análise do estado nutricional, procedeu-se a correção da idade das crianças em decorrência da prematuridade, considerando o peso com 40 semanas, correspondente à data prevista do nascimento. Devido a não totalidade de bebês com idade corrigida superior a 40 semanas no período correspondente e até 45 dias após 
a alta hospitalar, a avaliação do estado nutricional se deu a partir da terceira visita, período no qual todas as crianças já apresentavam idade corrigida maior que 40 semanas. Utilizaram-se então os índices de peso para a idade expressos em desvios-padrão (escore z) da curva de referência apresentada pela $\mathrm{WHO}$, as quais consideram crianças com o peso adequado aquelas com escore $Z \geq-2$, e desnutridas ou com baixo peso, as crianças com escore $Z<-2^{(6)}$.

Foram avaliadas as variáveis: a) Maternas (idade, escolaridade, situação conjugal e atividade); b) Neonatais (sexo, peso ao nascer, idade gestacional ao nascer e tipo de parto); c) Pós-natais (a prática ou não de aleitamento materno, o ganho de peso diário aos 15 e aos 45 dias após a alta hospitalar, e aos três e seis meses de vida, e o estado nutricional aos três e seis meses).

Os dados coletados e os resultados obtidos sobre o ganho ponderal diário e o estado nutricional foram categorizados e digitados em banco de dados no programa Microsoft Excel 2007. Para análise descritiva, utilizou-se frequência simples e proporções. Para verificar associação estatística entre variáveis maternas e neonatais e a prática ou não do aleitamento materno e o estado nutricional das crianças, foi aplicado o teste de hipótese Gamma, cujos níveis de significância foram considerados valor de $p<0,05$ com intervalo de confiança $95 \%$.

Para verificar a presença de correlação entre as variáveis maternas e neonatais e a prática do aleitamento materno com ganho ponderal diário, foi aplicado o teste de correlação ETA, o qual é apropriado para avaliar o grau de relacionamento entre duas variáveis, uma dependente medida numa escala de intervalo (g/dia) e outra independente com um número limitado de categorias. Os resultados do teste ETA podem variar entre - 1,00 e 1,00, passando pelo 0,00. Quanto mais próximo de 0,00 menor é a evidência de correlação entre as variáveis estudadas e os valores próximos a 1,00 ou - 1,00 sugerem alto grau de relacionamento, sendo que o sinal indica se a correlação é positiva ou negativa.

Cabe salientar que foram considerados como prematuros, os bebês nascidos com idade gestacional inferior a 37 semanas, e como tendo baixo peso ao nascer, aqueles com peso de nascimento menor que $2.500 \mathrm{~g}^{(6)}$. Além disso, em cada momento estudado, foram consideradas duas categorias de aleitamento materno: Aleitamento Materno Exclusivo (AME) nos casos de crianças que recebem apenas leite humano de forma direta (direto da mama) ou por ordenha, excetuando gotas, xaropes, suplementos minerais e outros medicamentos; e AM nos casos em que as crianças recebem o leite materno independentemente de receberem outros leites ou alimentos ${ }^{(1)}$.

O estudo foi desenvolvido em consonância com as diretrizes disciplinadas pela Resolução 196/96 do Conselho Nacional de Saúde e seu projeto foi aprovado pelo Comitê Permanente de Ética em Pesquisa envolvendo Seres Humanos da Universidade Estadual de Maringá (Parecer n 451/2008). Participaram do estudo somente os bebês cujas mães assinaram o termo de consentimento livre e esclarecido, autorizando a utilização das informações para fins de pesquisa.

\section{RESULTADOS}

No período de primeiro de maio a 31 de outubro de 2008, foram incluídos no PVBR 384 recém-nascidos e destes, 116 eram prematuros. Entretanto, devido a dados incompletos nos registros e desistência de algumas mães/famílias ao longo do desenvolvimento da pesquisa matricial, a amostra final para este estudo constituiu-se de 42 binômios (36,2\% do total).

A idade materna variou entre 17 e 40 anos, com média de 27 , sendo que $14,3 \%$ eram adolescentes, ou seja, com idade menor ou igual a 19 anos. A maioria das mulheres tinha companheiro $(90,5 \%)$, trabalhava fora $(61,9 \%)$ e apresentava tempo de estudo maior ou igual a oito anos (95,2\%). O parto cesáreo foi o mais frequente, representando $71,4 \%$ dos nascimentos. Quanto aos bebês, a maioria era do sexo masculino $(54,8 \%)$ e apresentava baixo peso ao nascer $(61,9 \%)$. O peso de nascimento variou entre $1255 \mathrm{~g}$ e $3440 \mathrm{~g}$, com média de $2393 \mathrm{~g}$, e a menor idade gestacional no nascimento foi de 30 semanas, sendo que $78,6 \%$ tinham entre 34 e 36 semanas. Todos apresentaram Apgar maior ou igual a sete no quinto minuto.

A prevalência do aleitamento materno (AM) e aleitamento materno exclusivo (AME) entre os bebês pré-termo estudados está descrita na Tabela 1.

Tabela 1 - Prevalência do aleitamento materno em bebês pré-termo aos 15 e 45 dias após a alta hospitalar, e aos três e seis meses de vida, no período 2008 - 2009. Maringá - PR

\begin{tabular}{ccccccccc}
\hline & \multicolumn{2}{c}{ AME } & \multicolumn{2}{c}{ AM } & \multicolumn{2}{c}{ Não AM } & \multicolumn{2}{c}{ TOTAL } \\
\hline & $\mathbf{n}$ & $\%$ & $\mathbf{n}$ & $\%$ & $\mathbf{n}$ & $\%$ & $\mathbf{N}$ & $\%$ \\
15 dias & 30 & 71,5 & 08 & 19,0 & 04 & 9,5 & 42 & 100,0 \\
45 dias & 19 & 45,2 & 15 & 35,7 & 08 & 19,1 & 42 & 100,0 \\
3 meses & 16 & 38,1 & 12 & 28,6 & 14 & 33,3 & 42 & 100,0 \\
6 meses & 15 & 35,7 & 02 & 4,8 & 25 & 59,5 & 42 & 100,0 \\
\hline
\end{tabular}

Notas:

AME = Aleitamento materno exclusivo;

$A M=$ aleitamento materno;

Não $A M=$ não recebem leite humano.

Pode-se perceber que a prática de AM estava presente em $90,5 \%$ (38) dos bebês aos 15 dias após alta hospitalar, em $81,0 \%$ (34) aos 45 dias, em 66,7\% (28) aos três meses e em $40,5 \%$ (17) aos seis meses de vida.

Vale salientar que o tipo de AM considerado em cada um dos períodos estudados foi aquele referido pela mãe durante as entrevistas e observado nas VD de acompanhamento dos bebês durante o estudo matricial, sendo considerada a ordem evolutiva AME, AM e não AM.

O ganho de peso diário dos bebês encontra-se descrito na Tabela 2.

Quanto ao estado nutricional das crianças, tanto no período da terceira como da quarta VD, correspondente aos três e aos seis meses de vida, 92,9\% apresentou escore $z$ dentro da faixa de normalidade ( $\geq-2$ ), sendo que não foram encontradas associações estatísticas entre as variáveis estudadas e o estado nutricional. 
Tabela 2 - Média do ganho ponderal diário entre bebês pré-termo conforme o tipo de aleitamento materno aos 15 e 45 dias após a alta hospitalar e aos três e seis meses de vida, no período 2008 - 2009. Maringá - PR

\begin{tabular}{lccc}
\hline & AME & AM & Não AM \\
\hline Ganho ponderal aos 15 dias (g/dia) & $\begin{array}{c}18,16 \\
(\mathrm{n}=30)\end{array}$ & $\begin{array}{c}13,37 \\
(\mathrm{n}=08)\end{array}$ & $\begin{array}{c}21,17 \\
(\mathrm{n}=04)\end{array}$ \\
Ganho ponderal aos 45 dias (g/dia) & $\begin{array}{c}39,17 \\
(\mathrm{n}=19)\end{array}$ & $\begin{array}{c}36,59 \\
(\mathrm{n}=15)\end{array}$ & $\begin{array}{c}31,14 \\
(\mathrm{n}=08)\end{array}$ \\
Ganho ponderal aos três meses (g/dia) & $\begin{array}{c}43,03 \\
(\mathrm{n}=16)\end{array}$ & $\begin{array}{c}39,49 \\
(\mathrm{n}=12)\end{array}$ & $\begin{array}{c}30,20 \\
(\mathrm{n}=14)\end{array}$ \\
Ganho ponderal aos seis meses (g/dia) & $\begin{array}{c}17,03 \\
(\mathrm{n}=15)\end{array}$ & $\begin{array}{c}18,58 \\
(\mathrm{n}=02)\end{array}$ & $\begin{array}{c}20,62 \\
(\mathrm{n}=25)\end{array}$ \\
\hline
\end{tabular}

Notas:

$A M E=$ Aleitamento materno exclusivo;

$A M=$ aleitamento materno;

Não $A M=$ não recebem leite humano.

Ao aplicar o teste estatístico GAMMA verificou-se que a prática do AM aos três meses estava associada tanto ao peso de nascimento $(p=0,01 /-0,623)$ quanto à idade gestacional de nascimento ( $p=0,01 /-0,583)$, demonstrando que quanto menor o peso e/ou menor a idade gestacional, maior a ocorrência de amamentação.

Embora a maioria dos bebês que estavam sendo amamentados aos 15 dias após a alta fossem crianças nascidas com baixo peso $(65,7 \%)$, não foi encontrada associação entre o peso ao nascer e o AM neste período. Em contrapartida, no sexto mês de vida houve uma queda de $64 \%$ na taxa de AM entre os bebês baixo peso, de modo que 34,6\% estavam sendo amamentados. Já nos bebês com peso de nascimento adequado, essa queda apresentou-se reduzida (38,4\%), com 50\% desses bebês mantidos em AM.

A taxa de AME apresentou valores consideráveis nos quatro períodos estudados, porém o AME não apresentou associação estatística com nenhumas das variáveis estudadas.

O teste ETA demonstrou correlação positiva à idade gestacional de nascimento e ao ganho ponderal aos 45 dias $(0,548)$, três meses $(0,302)$ e seis meses $(0,438)$, sendo estas correlações caracterizadas como forte, leve e moderada respectivamente, sugerindo que o nascimento mais próximo do termo está relacionado com a melhor evolução do ganho de peso diário. O peso ao nascer também apresentou correlação estatística leve com o ganho de peso aos 45 dias $(0,383)$, três meses $(0,389)$ e seis meses $(0,313)$, demonstrando que quanto maior o peso ao nascer, maior é o ganho ponderal nesses períodos.

A idade materna demonstrou relacionar-se forte e positivamente com o ganho ponderal nos quatro períodos estudados (15 dias: 0,629; 45 dias: 0,791; três meses: 0,579 e seis meses: $0,602)$, denotando que quanto maior a idade materna, melhor é o processo de ganho ponderal do bebê. Entretanto, quando a idade materna foi categorizada em mães adultas e mães adolescentes, não foi encontrada correlação entre as variáveis.

A prática do AM aos 15 dias apresentou correlação leve e positiva com o ganho ponderal aos seis meses (0,333). Esta foi a única correlação encontrada entre a amamentação e o ganho ponderal.

\section{DISCUSSÃO}

As taxas de AM e AME encontradas neste estudo apresentaram valores expressivos quando comparadas à prevalência de AM no conjunto das capitais brasileiras e no Distrito Federal, nas quais se constatou que, no ano de 2008 a duração mediana do AME foi de 54,1 dias (1,8 meses) e a duração mediana do AM foi de 341,6 dias $(11,2 \text { meses })^{(7)}$. Destaca-se ainda que a população do estudo atual constituiu-se de bebês prematuros, contexto este específico e no qual o processo de amamentação configura-se como um desafio, já que estes bebês apresentam imaturidade fisiológica e neurológica, que predispõe os mesmos a permanecerem em estado de alerta por períodos muito curtos, em função da hipotonia muscular e hiper-reatividade aos estímulos do meio ambiente ${ }^{(8)}$, fatores estes que interferem na iniciação e no estabelecimento da amamentação.

A falta de contato precoce entre mãe-filho e a ausência da amamentação na sala de parto inibem o pico da produção láctea materna, quadro que pode ainda ser agravado pela longa permanência do bebê na UTIN ${ }^{(4)}$, e, mesmo que a mãe siga as orientações recebidas da equipe no sentido da manutenção da lactação, muitas vezes esta não consegue evitar a diminuição da produção do leite ${ }^{(9)}$. Dessa maneira, bebês nascidos prematuros, além de tratamento e cuidados especiais, necessitam de maior incentivo e apoio à prática do AM, para que uma melhor qualidade de assistência e de vida seja assegurada $^{(9)}$.

Embora as taxas de AM e AME tenham se mostrado elevadas entre os bebês estudados, entre aqueles que nasceram com baixo peso, ainda que sem a comprovação de uma associação estatística, a queda da prevalência do AM entre os 15 dias após a alta e os seis meses de vida foi maior entre os bebês de baixo peso, quando comparados com os nascidos com peso adequado. Dessa forma, a atenção voltada à promoção e ao estabelecimento do AM nessa população especial deve estender-se para além do período de hospitalização e do pós-alta imediato, voltando-se para as dificuldades do binômio durante sua adaptação à rotina domiciliar, bem como no enfrentamento das possíveis intercorrências que possam surgir neste período ${ }^{(10)}$.

No presente estudo, verificou-se que a prática do AM estava associada tanto ao peso de nascimento quanto à idade gestacional, demonstrando que quanto menor o peso e/ou menor a idade gestacional, maior a ocorrência de amamentação. Isso é um fator positivo, visto já ter sido identificado que bebês prematuros e nascidos com baixo peso que recebem leite materno, permanecem menos tempo internados, e têm menor perda de peso do que aqueles que são alimentados por fórmula láctea ${ }^{(4)}$.

Resultados semelhantes foram encontrados em pesquisa que identificou associação estatística entre peso ao nascer, idade gestacional e duração do internamento do prematuro com a duração de $\mathrm{AME}^{(11)}$. Outro estudo, por sua vez, 
identificou correlação negativa entre peso ao nascer, idade gestacional e desmame parcial, sendo que quanto menor o peso e a idade gestacional, mais tardia foi a introdução da mamadeira ${ }^{(12)}$.

Identificou-se que os bebês nos primeiros quinze dias não atingiram o ganho ponderal de 25 a $30 \mathrm{~g}$ esperados por dia no primeiro trimestre ${ }^{(13)}$. Cabe salientar que a necessidade calórica para o recém-nascido pré-termo é de 120-130Kcal/kg/ dia, para um ganho ponderal esperado de $15 \mathrm{~g} / \mathrm{dia}$, tendo o colostro um valor calórico estimado de 58 calorias $/ 100 \mathrm{~mL}^{(3)}$.

Contudo, ao final do primeiro trimestre, os bebês atingiram média de ganho ponderal de 43,03g/dia entre os com AME, 39,49g/dia nos com AM e de 30,20g/dia entre aqueles não $\mathrm{AM}$, ultrapassando o peso diário esperado. Em relação ao segundo trimestre, esperava-se que os bebês tivessem um ganho ponderal de 20 a $25 \mathrm{~g} / \mathrm{dia}$, porém somente os bebês que não receberam o leite materno atingiram o ganho de 20,62g/dia; os demais atingiram a medida de 17,03g/dia (AME) e 18,58g/dia (AM).

Pesquisa realizada em Taiwan, que monitorou por sete dias a oferta de leite e o peso de 14 bebês com idade gestacional de 27 a 36 semanas, sendo quatro de muito baixo peso e quatro de extremo baixo peso (peso corporal de 680 a 3050g), evidenciou que todos receberam AM e tiveram ganho de peso ponderal adequado. A média da Kcal do leite oferecido foi de $0,67 \mathrm{Kcal} / \mathrm{mL}$ e o início da nutrição enteral em todos os RN ocorreu no $15^{\circ}$ dia de vida ${ }^{(14)}$.

A prática maior do AM em crianças nascidas com baixo peso e menor idade gestacional pode estar relacionada ao fato da mãe receber maior apoio à amamentação, visto que o bebê é submetido a longos períodos de internação e durante o estabelecimento do aleitamento, o binômio contar com o apoio constante dos membros da equipe de saúde que atuam como facilitadores deste processo ${ }^{(15)}$. Um exemplo disso é o método Canguru. Em estudo realizado com bebês pré-termo que permaneceram internados na Enfermaria Canguru, encontrou-se alto índice de AME após a alta hospitalar ${ }^{(4)}$, considerando que este método é uma ótima estratégia de estímulo à prática da amamentação e que a assistência recebida pela mulher durante o processo de parto, nascimento e internação conjunta influencia positivamente a amamentação ${ }^{(16)}$.

Por outro lado, a utilização de fórmulas supõe, de certa forma, a predisposição materna ao desmame precoce, já que a utilização deste tipo de alimentação na hospitalização faz com que as mães se sintam mais confortáveis para o desmame, fato que as leva a deixar de oferecer ao filho o seu próprio leite e, por conseguinte todas as propriedades benéficas do leite materno ${ }^{(17)}$.

A correlação entre o maior ganho ponderal e a maior idade materna esteve presente nos quatro momentos estudados, embora não tenha sido encontrada associação entre a idade materna e a prática do AM. Outros estudos, no entanto, têm identificado que a prevalência de AME é maior nas mulheres mais velhas ${ }^{(9,18)}$.

Esses dados sugerem que, quanto maior a idade materna, maior será a ocorrência de amamentação, e consequentemente, maior o ganho ponderal, principalmente no início da vida do bebê ${ }^{(9,19)}$. Tais achados podem ser justificados devido a uma maior capacidade de compreensão e maturidade das mulheres adultas em relação à importância da prática do AM, e ao maior comprometimento advindo dessa compreensão. Além disso, a experiência prévia de AM pode ser um fator colaborador para a prática atual de alimentação do filho ${ }^{(20)}$.

Além da idade, a presença de um companheiro, relatada pela maior parte das mães deste estudo, embora não tenha apresentado correlação com significância estatística, pode ser considerado ponto positivo para a prática do AM, visto que estudo realizado com lactentes de até dois anos em Campinas mostrou que as mulheres sem companheiro têm risco 17\% maior de amamentar seus filhos por menos tempo ${ }^{(21)}$.

Neste estudo, a proporção de mães com escolaridade maior ou igual a oito anos abrangeu quase a totalidade da amostra, o que pode ter influenciado os resultados obtidos com relação à prática de $\mathrm{AM}$ e ao ganho ponderal observados. Tais achados corroboram os resultados de outros estudos que concluíram que mulheres com escolaridade mais elevada apresentam maiores frequências de $\mathrm{AME}^{(9,22)}$, enquanto que a baixa escolaridade e a atividade fora do lar estão associadas à introdução precoce de alimentos ${ }^{(23)}$, interferindo no crescimento saudável e adequado da criança.

Para além da influência das características maternas na manutenção e estabelecimento do AM, estudo que abordou as experiências vivenciadas pelas mães de prematuros constatou que a dificuldade de manter a produção láctea durante a hospitalização do bebê na UTIN pode colocar em risco o estabelecimento do AM nestes bebês ${ }^{(24)}$. Verificou ainda que, de acordo com as mães, mesmo quando elas seguem as orientações recebidas dos profissionais de saúde, não conseguem evitar a diminuição da produção láctea e apontam a ausência da sucção do recém-nascido ao peito como a principal razão para isto ocorrer, embora reconheçam a influência do contexto geral, caracterizado pelas condições físicas do filho e também a seu próprio estado físico mental(24).

Reforçando estes achados, estudo de revisão sobre os fatores associados à manutenção do aleitamento materno de recém-nascidos pré-termo, destacou como fatores dificultadores a prematuridade, a fragilidade do RN e a dificuldade de sucção no peito materno, as sequelas neonatais; o tempo de internação e de separação da mãe e filho. Por outro lado, dentre os fatores que facilitaram a manutenção da AM destacou a realização da ordenha mamária manual ou elétrica, o desenvolvimento do vínculo entre a mãe e o bebê, apoio dos profissionais de saúde e dos pais para o $\mathrm{AM}$ e o início do AM durante a internação na UTIN ${ }^{(22)}$.

No presente estudo, a correlação observada entre o maior ganho ponderal e o nascimento mais próximo do termo, assim como em relação ao maior peso ao nascer, podem estar relacionadas com o fato dos bebês apresentarem melhores condições de crescimento desde o ambiente intra-útero, com maior maturidade fisiológica e reservas energéticas para o enfrentamento dos primeiros dias de vida. No entanto, é importante lembrar que a correlação encontrada foi leve, e ainda se contrapõe a achados de outro estudo realizado com 3172 crianças nascidas a termo, que demonstrou que o peso de 
nascimento tem relação negativa com o ganho de peso nos primeiros seis meses ${ }^{(25)}$.

A correlação entre a prática do AM aos 15 dias após a alta hospitalar e o maior ganho ponderal aos seis meses de vida corrobora as conclusões de outro estudo em que foi identificado que lactentes amamentados com leite materno crescem mais rapidamente no começo da vida, quando comparados aos lactentes alimentados com fórmulas lácteas ${ }^{(19)}$. Tais crianças (com predomínio do AM) apresentam melhores velocidades de crescimento nos primeiros meses, porém a velocidade de crescimento a partir do sétimo mês de vida torna-se maior entre as crianças alimentadas apenas com fórmulas ${ }^{(19)}$. $\mathrm{O}$ fato de ter sido incluído no referido estudo tanto bebês prematuros quanto a termo impede uma comparação mais linear entre os resultados.

Ainda no que tange ao ganho ponderal, um estudo alemão realizado com lactentes aos nove meses de vida constatou que crianças que não foram amamentadas ou o foram por menos de quatro meses, apresentaram desde o nascimento, score $z$ de peso para comprimento menor do que aquelas crianças que receberam leite materno por seis meses ou mais ${ }^{(26)}$.

\section{CONSIDERAÇÕES FINAIS}

Não obstante o fato de haver limitações ao estabelecimento do AM na população de prematuros, fica cada vez mais evidenciada a sua viabilidade e importância, dados os benefícios imensuráveis no âmbito biológico e psicossocial tanto para a criança, quanto para a mãe e a família.

São poucos os estudos que contemplem a temática do aleitamento materno no contexto específico dos bebês prematuros. Seus benefícios com relação às propriedades imunológicas e nutritivas já estão mais do que comprovados na literatura médica; entretanto, ainda não se sabe ao certo o quanto essa prática é capaz de favorecer o processo de crescimento e de ganho ponderal dessas crianças.

Conhecer quais os fatores que favorecem a prática da amamentação, assim como aqueles que interferem neste processo, é imprescindível para o planejamento de ações voltadas a tal clientela, de modo a propiciar que os benefícios desta prática possam ser usufruídos plenamente pelo binômio, pela família e pela comunidade. Ser enfermeiro abrange não só atender as demandas de seus clientes, mas também prever possíveis necessidades e dificuldades, e dessa maneira, buscar estratégias que permitam contorná-las de forma efetiva, com apoio, orientação e escuta compreensiva.

É importante salientar que, embora este estudo tenha encontrado resultados importantes, o pequeno número amostral constitui uma limitação, pois impossibilitou testes estatísticos mais aprofundados, o que aponta a necessidade de realização de novas pesquisas com o intuito de identificar outros fatores relacionados com a prática do aleitamento materno entre pré-termos e suas repercussões sobre o crescimento, em especial, sobre o ganho ponderal dessas crianças.

\section{REFERÊNCIAS}

1. Ministério da Saúde (BR). Saúde da criança: nutrição infantil: aleitamento materno e alimentação complementar. Brasília, DF; 2009.

2. Vandenplas $Y$, Veereman-Wauters G, Greef E, Peeters S, Casteels A, Mahler T, et al. Probióticos e prebióticos na prevenção e no tratamento de doenças em lactentes e crianças. J Pediatr (Rio J) [Internet]. 2011 [acesso em 05 setembro de 2012];87(4):292-300. Disponível em: http://www.scielo.br/scielo.php?pid = S0021$-75572011000400004 \&$ script $=$ sci_arttext

3. Tamez RN, Silva MJP. Enfermagem na UTI neonatal: assistência ao recém-nascido de alto risco. 4.ed. Rio de Janeiro (RJ): Guanabara-Koogan; 2009.

4. Alves AML, Silva EHAA, Oliveira AC. Desmame precoce em prematuros participantes do método mãe-canguru. Rev Soc Bras Fonoaudiol [Internet]. 2007 [acesso em 04 de junho de 2014];12(1):23-8. Disponível em: http://www.scielo.br/scielo.php?script=sci_arttext\&pid $=$ S1516-80342007000100006

5. Use and interpretation of anthropometric indicators of nutritional status. Bull World Health Organ [Internet]. 1986 [cited 2014 June 04];64(6):929-41. Available from: http:// www.ncbi.nlm.nih.gov/pubmed/3493862

6. Ministério da Saúde (BR). Secretaria de Atenção a Saúde. Departamento de Ações Programáticas e Estratégicas.
II Pesquisa de Prevalência de Aleitamento Materno nas Capitais Brasileiras e Distrito Federal. Brasília (DF); Ministério da Saúde; 2009.

7. Hayakawa LM, Schmidt KT, Rossetto EG, Souza SNDH, Bengozi TM. Incidência de reinternação de prematuros com muito baixo peso nascidos em um hospital universitário. Esc Anna Nery Rev Enferm [Internet]. 2010 [acesso em 04 de junho de 2014];14(2):324-9. Disponível em: http://www.scielo.br/scielo.php?script=sci_arttext\&pid $=\mathrm{S} 1414-81452010000200016$

8. Sassá AH, Schmidt KT, Teston EF, Marcon SS. Growth and feeding practices to newborn with very low weight along the first year of life - descriptive study. Online Braz J Nurs [Internet]. 2010 [Cited 2010 November 23]; 9(2). Available from: http://www.objnursing.uff.br/index.php/ nursing/article/view/3074

9. Pereira RSV, Oliveira MIC, Andrade CLT, Brito AS. Fatores associados ao aleitamento materno exclusivo: o papel do cuidado na atenção básica. Cad Saúde Pública [Internet]. 2010 [acesso em 02 de junho de 2014];26(12):2343-54. Disponível em: http://www.redeblh.fiocruz.br/media/artigocadsp10.pdf

10. Sassá AH, Marcon SS. Avaliação de famílias de bebês nascidos com muito baixo peso durante o cuidado domiciliar. Texto \& Contexto Enferm [Internet]. 2013 [acesso 
em 06 de junho de 2014];22(2):442-51. Disponível em: http://www.scielo.br/pdf/tce/v22n2/v22n2a21.pdf

11. Oliveira MMB, Thomson Z, Vannuchi MTO, Matsuo T. Feeding patterns of Brazilian preterm infants during the first 6 months of life, Londrina, Paraná, Brasil. J Hum Lact [Internet]. 2007 [cited 2014 June 06];23(3):269-74. Available from: http://www.ncbi.nlm.nih.gov/pubmed/17666537

12. Silva SMS, Segre CAM. Fatores que influenciam o desmame no recém-nascido prematuro. Rev Bras Crescimento Desenvolv Hum [Internet]. 2010 [acesso em 29 de maio de 2014];20(2):291-301. Disponível em: http://pepsic.bvsalud.org/pdf/rbcdh/v20n2/12.pdf

13. Ministério da Saúde (BR). Atenção à saúde do recém-nascido: guia para os profissionais de saúde. Cuidados com o recém-nascido pré-termo [Internet]. Brasília (DF): Ministério da Saúde; 2011 [acesso em 04 de junho de 2014]. Disponível em: http://www.redeblh.fiocruz.br/ media/arn v4.pdf

14. Lin HY, Hsieh HY, Chen HH, Chiu HY, Lin HC, Su BH. Efficacy of creamatocrit technique in evaluation of premature infants fed with breast milk. Pediatr Neonatol [Internet]. 2011 [cited 2014 June 04];52(3):130-4. Available from: http://www.ncbi.nlm.nih.gov/pubmed/21703553

15. Silva CC, Paula GR, Peixoto MKAV, Oliveira NES, Santos LF, Oliveira LMAC. Assistência de enfermagem a familiares de recém-nascidos em unidade de terapia intensiva neonatal: revisão integrativa. Ciênc Cuid Saúde [Internet]. 2013 [acesso em 30 de maio de 2014];12(4):804-12. Disponível em: http://periodicos.uem.br/ojs/index.php/ CiencCuidSaude/article/view/22550/pdf 102

16. Narchi NZ, Fernandes RAQ, Dias LA, Novais DH. Variáveis que influenciam a manutenção do aleitamento materno exclusivo. Rev Esc Enferm USP [Internet]. 2009 [acesso em 04 de junho de 2014];43(1):87-94. Disponível em: http://www.scielo.br/pdf/reeusp/v43n1/11.pdf

17. Aimone A, Rovet J, Ward W, Jefferies A, Campbell DM, Asztalos $\mathrm{E}$, et al. Growth and body composition of human milk-fed premature infants provided with extra energy and nutrients early after hospital discharge: 1-year follow-up. J Pediatr Gastroenterol Nutr [Internet]. 2009 [cited 2014 June 04]; 49(4):456-66. Available from: http://www.ncbi. nlm.nih.gov/pubmed/19633578

18. Araújo NL, Lima LHO, Oliveira EAR, Carvalho ES, Duailibe FT, Formiga LMF. Alimentação infantil e fatores relacionados ao aleitamento materno. Rev RENE [Internet]. 2013 [acesso em 04 de junho de 2014];14(6):1064-72. Disponível em: http://www.revistarene.ufc.br/revista/index.php/revista/article/view/1309/pdf
19. Spyrides MHC, Struchiner CJ, Barbosa MTS, Kac G. $\mathrm{O}$ efeito das práticas alimentares sobre o crescimento infantil. Rev Bras Saúde Mater Infant [Internet]. 2005 [acesso em 04 de junho de 2014];5(2):145-53. Disponível em: http://www.scielo.br/scielo.php?pid=s1519$-38292005000200002 \&$ script $=$ sci arttext

20. Fujimori E, Nakamura E, Gomes MM, Jesus LA, Rezende MA. Aspectos relacionados ao estabelecimento e à manutenção do aleitamento materno exclusivo na perspectiva de mulheres atendidas em uma unidade básica de saúde. Interface Comun Saúde Educ [Internet]. 2010 [acesso em 06 junho de 2014];14(33):315-27. Disponível em: http:// www.scielo.br/pdf/icse/v14n33/a07v14n33.pdf

21. Bernardi JLD, Jordão RE, Barros Filho AA. Fatores associados à duração mediana do aleitamento materno em lactentes nascidos em município do estado de São Paulo. Rev Nutr [Internet]. 2009 [acesso em 05 de junho de 2014];22(6):867-78. Disponível em: http://www.scielo. $\mathrm{br} / \mathrm{pdf} / \mathrm{rn} / \mathrm{v} 22 \mathrm{n} 6 / \mathrm{v} 22 \mathrm{n} 6 \mathrm{a} 08 . \mathrm{pdf}$

22. Rodrigues AP, Martins EL, Trojahn TC, Padoin SMM, Paula CC, Tronco CS. Manutenção do aleitamento materno de recém-nascidos pré-termo: revisão integrativa da literatura. Rev Eletrônica Enferm [Internet]. 2013 [acesso em 05 de junho de 2014];15(1):253-64. Disponível em: http:// www.fen.ufg.br/fen_revista/v15/n1/pdf/v15n1a29.pdf

23. Tarranti M, Fong DYT, Wu KM, Lee ILY, Wong EMY, Sham A, et al. Breastfeeding and weaning practices among Hong Kong mothers: a prospective study. BMC Pregnancy Childbirth [Internet]. 2010 [cited 2014 Nune 05];10:27. Available from: http://www.ncbi.nlm.nih.gov/pubmed/20509959

24. Silva RV, Silva IA. A vivência de mães de recém-nascidos prematuros no processo de lactação e amamentação. Esc Anna Nery Rev Enferm [Internet]. 2009 [acesso em 03 de junho de 2014];13(1):108-15. Disponível em: http:// www.scielo.br/pdf/ean/v13n1/v13n1a15

25. Longo GZ, Souza JMP, Souza SB, Szarfarc SC. Crescimento de crianças até seis meses de idade, segundo categorias de aleitamento. Rev Bras Saúde Matern Infant [Internet]. 2005 [acesso em 03 de junho de 2014];5(1):109-18. Disponível em: http://www.scielo.br/scielo.php?pid=S1519$-38292005000100014 \&$ script $=$ sci arttext

26. Rebhan B, Kohlhuber $M$, Schwegler $U$, Fromme $H$, Abou-Dakn M, Koletzko BV. Breastfeeding duration and exclusivity associated with infants' health and growth: data from a prospective cohort study in Bavaria, Germany. Acta Pediatr [Internet]. 2009 [cited 2014 June 04];98(6):974-80. Avaliable from: http://www.ncbi.nlm. nih.gov/pubmed/19484835 